\title{
A novel sweetpotato bZIP transcription factor gene, IbbZIP1, is involved in salt and drought tolerance in transgenic Arabidopsis
}

\author{
Chen Kang ${ }^{1} \cdot$ Hong Zhai ${ }^{1}$ Shaozhen $\mathrm{He}^{1} \cdot$ Ning Zhao $^{1} \cdot$ Qingchang Liu $^{1}$
}

Received: 10 April 2019 / Accepted: 4 June 2019 / Published online: 10 June 2019

(c) The Author(s) 2019

\begin{abstract}
Key message The overexpression of IbbZIPI leads to a significant upregulation of abiotic-related genes, suggesting that IbbZIP1 gene confers salt and drought tolerance in transgenic Arabidopsis.

Abstract Basic region/leucine zipper motif (bZIP) transcription factors regulate flower development, seed maturation, pathogen defense, and stress signaling in plants. Here, we cloned a novel bZIP transcription factor gene, named IbbZIPI, from sweetpotato [Ipomoea batatas (L.) Lam.] line HVB-3. The full length of IbbZIPI exhibited transactivation activity in yeast. The expression of IbbZIP1 in sweetpotato was strongly induced by $\mathrm{NaCl}$, PEG6000, and abscisic acid (ABA). Its overexpression in Arabidopsis significantly enhanced salt and drought tolerance. Under salt and drought stresses, the transgenic Arabidopsis plants showed significant upregulation of the genes involved in ABA and proline biosynthesis and reactive oxygen species scavenging system, significant increase of ABA and proline contents and superoxide dismutase activity and significant decrease of $\mathrm{H}_{2} \mathrm{O}_{2}$ content. These results demonstrate that the IbbZIPI gene confers salt and drought tolerance in transgenic Arabidopsis. This study provides a novel bZIP gene for improving the tolerance of sweetpotato and other plants to abiotic stresses.
\end{abstract}

Keywords Arabidopsis $\cdot$ IbbZIP1 $\cdot$ Salt and drought tolerance $\cdot$ Sweetpotato

$\begin{array}{ll}\text { Abbreviations } \\ \text { bZIP } & \text { Basic region/leucine zipper motif } \\ \text { EST } & \text { Expressed sequence tag } \\ \text { RACE } & \text { Rapid amplification of cDNA ends } \\ p \text { I } & \text { Isoelectric point } \\ \text { ABA } & \text { Abscisic acid } \\ \text { SOD } & \text { Superoxide dismutase } \\ \text { ROS } & \text { Reactive oxygen species } \\ \text { PPT } & \text { Phosphinothricin }\end{array}$

Communicated by Sang-Soo Kwak.

Electronic supplementary material The online version of this article (https://doi.org/10.1007/s00299-019-02441-x) contains supplementary material, which is available to authorized users.

Qingchang Liu

liuqc@cau.edu.cn

1 Key Laboratory of Sweetpotato Biology and Biotechnology, Ministry of Agriculture/Beijing Key Laboratory of Crop Genetic Improvement/Laboratory of Crop Heterosis and Utilization, Ministry of Education, College of Agronomy and Biotechnology, China Agricultural University, Beijing 100193, China

\section{Introduction}

Salinity and drought seriously affect the productivity of agricultural crops in the world (Munns and Tester 2008; Yang et al. 2010; Zhao et al. 2013). Developing crops tolerant to salinity and drought is becoming important. Plants adapt to salinity and drought stresses by developing a variety of mechanisms, such as growth and development regulation, osmotic adjustment, detoxification, and ion homeostasis (Bohnert et al. 1995; Zhu 2001, 2002).

The basic region/leucine zipper motif (bZIP) proteins compose a large family of transcription factors in higher plants. The bZIP members have been reported to regulate flower development, seed maturation, pathogen defense and stress, light, hormone and sugar signaling pathways (Jakoby et al. 2002; Lindemose et al. 2013). Several bZIP genes have been found to confer the tolerance to abiotic stresses in some plant species. In Arabidopsis, AtABF3, AtbZIP24, and $A t b Z I P 1$ are positive regulators of plant tolerance to abiotic stresses (Kim et al. 2004; Yang et al. 2009; Sun et al. 2012). In rice, OsbZIP23, OsbZIP72, OsABF1/OsbZIP12, OsABF2/OsbZIP46, and OsbZIP71 increased the tolerance to abiotic stresses (Xiang et al. 2008; Lu et al. 2009; Hossain 
et al. 2010a, b; Liu et al. 2014a, b, c, d), but OsbZIP52 negatively regulates responses to cold and drought (Liu et al. 2012). GmbZIP44, GmbZIP62, and GmbZIP78 conferred salt and freezing tolerance in transgenic Arabidopsis (Liao et al. 2008). GmbZIPI increased the tolerance to salinity, cold temperature and drought in transgenic Arabidopsis and improved drought tolerance in transgenic wheat (Gao et al. 2011a, b). ZmbZIP72 conferred salt and drought tolerance in transgenic Arabidopsis (Ying et al. 2012) and ZmABP9 enhanced salt and drought tolerance in transgenic cotton (Wang et al. 2017).

Sweetpotato is an important food crop worldwide (Zang et al. 2009). Its productivity is often limited by salinity and drought stresses (Liu et al. 2014a). Gene engineering is an alternative approach for improving abiotic stresses' tolerance in sweetpotato (Kim et al. 2012, 2013, 2014; Liu et al. 2014a, b, c, 2015; Wang et al. 2016a, b, c; Zhai et al. 2015; Li et al. 2017). To date, the bZIP family has not been reported in sweetpotato. Here, a novel bZIP transcription factor gene, named IbbZIPI, was isolated from sweetpotato and found to be involved in salt and drought tolerance in transgenic Arabidopsis.

\section{Materials and methods}

\section{Plant materials}

Sweetpotato line HVB-3 was used to isolate the IbbZIPI gene in this study. Its transcriptome sequencing was conducted by Li et al. (2017), from which one expressed sequence tag (EST) was obtained. Arabidopsis thaliana (Columbia-0, WT) was employed to analyze the function of IbbZIPI.

\section{Cloning and analysis of $I b b Z I P 1$ and its promoter}

Freshly-harvested storage roots of HVB-3 were used to extract total RNA. The first-strand cDNA was obtained using PrimeScript ${ }^{\mathrm{TM}}$ II 1st Strand cDNA Synthesis Kit (TaKaRa, Beijing, China). A rapid amplification of cDNA ends (RACE) procedure was applied to amplify the fulllength cDNA of IbbZIPI with specific primers (Supplementary Table S1). Genomic DNA extracted from in vitrogrown plants was used to amplify the genomic sequence of IbbZIPI (Wang et al. 2016a). Its promoter was cloned with Universal GenomeWalker 2.0 Kit (TaKaRa, Dalian, China). The specific primers listed in Supplementary Table S1 were employed.

The IbbZIPI cDNA analysis was performed online (https ://blast.ncbi.nlm.nih.gov/Blast.cgi). The ORF Finder (https ://www.ncbi.nlm.nih.gov/orffinder/) was used to predict its open-reading frame (ORF). The phylogenetic analysis was conducted with the DNAMAN software (Lynnon Biosoft, Quebec, Canada). Exon-intron structure was constructed using Splign tool (https://www.ncbi.nlm.nih.gov/sutils/splig $\mathrm{n} /$ splign.cgi?textpage $=$ online \&level $=$ form) and compared with the Atlg58110 gene (http://www.arabidopsis.org/). The molecular weight and theoretical isoelectric point $(p \mathrm{I})$ of the IbbZIP1 protein were analyzed online (http://web.expas y.org/compute_pi/). PlantCARE (http://bioinformatics.psb. ugent.be/webtools/plantcare/html/) was employed to identify the $c i s$-acting regulatory elements in the promoter region of IbbZIPI.

\section{Expression analysis of IbbZIP1 in sweetpotato}

Storage root, stem, and leaf tissues of HVB-3 grown for 100 days in a field were used to isolate total RNA. The expression of IbbZIPI was analyzed by quantitative realtime PCR (qRT-PCR) with the specific primers of IbbZIPI (Supplementary Table S1) and Ibactin (AY905538) as an internal control based on the method of Liu et al. (2014a). Comparative $C_{\mathrm{T}}$ method was employed to quantify the gene expression (Schmittgen and Livak 2008). Furthermore, the HVB-3 plants cultured for 4 weeks on the basal Murashige and Skoog (MS) medium were transferred to liquid MS medium with $\mathrm{H}_{2} \mathrm{O}$ (control), $200 \mathrm{mM} \mathrm{NaCl}, 20 \%$ PEG6000 and $100 \mu \mathrm{M} \mathrm{ABA}$, respectively, and sampled at $0,2,4,6$, 12,24 , and $48 \mathrm{~h}$ after treatment for analyzing the expression of IbbZIPI.

\section{Transactivation assay of IbbZIP1 in yeast}

Transactivation assay of the IbbZIP1 protein in yeast (Saccharomyces cerevisiae) was done as described by Jiang et al. (2014). Its encoding region obtained with specific primers (Supplementary Table S1) was integrated into the vector pGBKT7 (pBD). Expression vector pBD-IbbZIP1, pGAL4 (as positive vector) and $\mathrm{pBD}$ (as negative vector) were transferred into the yeast strain AH109, respectively. The transactivation activity was determined according to the method of Wang et al. (2016a).

\section{Production of the transgenic Arabidopsis plants}

The overexpression vector $\mathrm{pC} 3301-121-I b b Z I P 1$ was constructed by inserting 35S-IbbZIPI-NOS into pCAMBIA3301 at HindIII and EcoRI and then transferred into the Agrobacterium tumefaciens strain GV3101. The dipping flower method was applied to transform Arabidopsis (Clough and Bent 1998). The transgenic Arabidopsis plants were determined by histochemical GUS assay (Jefferson et al. 1987) and PCR analysis with $35 \mathrm{~S}$ forward and IbbZIPI-specific reverse primers (Supplementary Table $S 1$ ). 


\section{Assay for salt and drought tolerance}

Transgenic Arabidopsis $\mathrm{T}_{3}$ and WT seedlings were treated on MS medium containing $200 \mathrm{mM} \mathrm{NaCl}$ or $300 \mathrm{mM}$ mannitol under $13 \mathrm{~h}$ day-light at $54 \mu \mathrm{M} / \mathrm{m}^{2} / \mathrm{s}$ and $22{ }^{\circ} \mathrm{C}$. After 2 weeks, their root length and fresh weight were investigated. Furthermore, the $\mathrm{T}_{3}$ and WT seedlings were planted for 2 weeks in pots with a soil, vermiculite, and humus mixture $(1: 1: 1, \mathrm{v} / \mathrm{v} / \mathrm{v})$ and each pot was irrigated with a $33 \mathrm{~mL}$ of $300 \mathrm{mM} \mathrm{NaCl}$ solution once every 2 days for 2 weeks, or stressed by drought for 4 weeks and re-watered for 2 days. The contents of abscisic acid (ABA) and proline and the activity of superoxide dismutase (SOD) in the $\mathrm{T}_{3}$ and WT plants treated in pots for 4 weeks under no stresses, 1 week under $300 \mathrm{mM} \mathrm{NaCl}$ stress or 2 weeks under drought stress were determined as described by Gao et al. (2011a). The $\mathrm{H}_{2} \mathrm{O}_{2}$ content was measured with $\mathrm{H}_{2} \mathrm{O}_{2}$ Assay Kit (Comin Biotechnology Co., Ltd. Suzhou, China). Twenty-seven plants in three pots with nine plants per pot were treated for each line.

\section{Assay for ABA sensitivity}

For ABA sensitivity assay, the transgenic Arabidopsis and WT seeds (50 seeds for each line) were sown on MS medium with $0,0.5$, and $1 \mu \mathrm{M}$ ABA, respectively, under $13 \mathrm{~h}$ day-light at $54 \mu \mathrm{M} / \mathrm{m}^{2} / \mathrm{s}$ and $22{ }^{\circ} \mathrm{C}$. After 1 week, their germination rate and cotyledon opening and greening rate were investigated.

\section{Expression analysis of the related genes}

The $\mathrm{T}_{3}$ and WT plants potted for 4 weeks without stress, 1 week stressed with $300 \mathrm{mM} \mathrm{NaCl}$ or 2 weeks stressed by drought were employed to analyze the expression of the genes involved in ABA and proline biosynthesis and reactive oxygen species (ROS) scavenging system using qRT-PCR protocols of Liu et al. (2014a). The specific primers of Atactin (internal control, NM112764) and the related genes were listed in Supplementary Table S1.

\section{Statistical analysis}

All experiments were performed with three biological replicates. Data presented as the mean $\pm \mathrm{SE}$ were analyzed by Student's $t$ test (two-tailed analysis) at $P<0.05(*)$ and $P<0.01$ $(* *)$.

\section{Results}

\section{Cloning and sequence analysis of IbbZIP1 and its promoter}

The IbbZIPl gene was cloned from sweetpotato line HVB-3. The cDNA sequence was $1757 \mathrm{bp}$ in length and contained an 1080 bp ORF encoding a 359-aa polypeptide with a molecular weight of $41.26 \mathrm{kDa}$ and a predicted $p \mathrm{I}$ of 7.89 . The IbbZIP1 protein was subjected to phylogenetic analysis together with 44 Arabidopsis bZIP proteins belonging to 10 groups (Jakoby et al. 2002). The results revealed that IbbZIP1 belonged to group E of the bZIP family and had a close relationship with At1g58110 (Fig. 1). IbbZIP1 contained one bZIP domain (Fig. 2a). The 1946 bp genomic DNA of IbbZIP 1 contained 4 exons and 3 introns (Fig. 2b). Its promoter region ( $2056 \mathrm{bp}$ ) had the stress-responsive cis-acting regulatory elements, such as HSE, MBS, TCA, GARE, TGA, ERE, and ABRE (Supplementary Fig. S1; Supplementary Table S2).

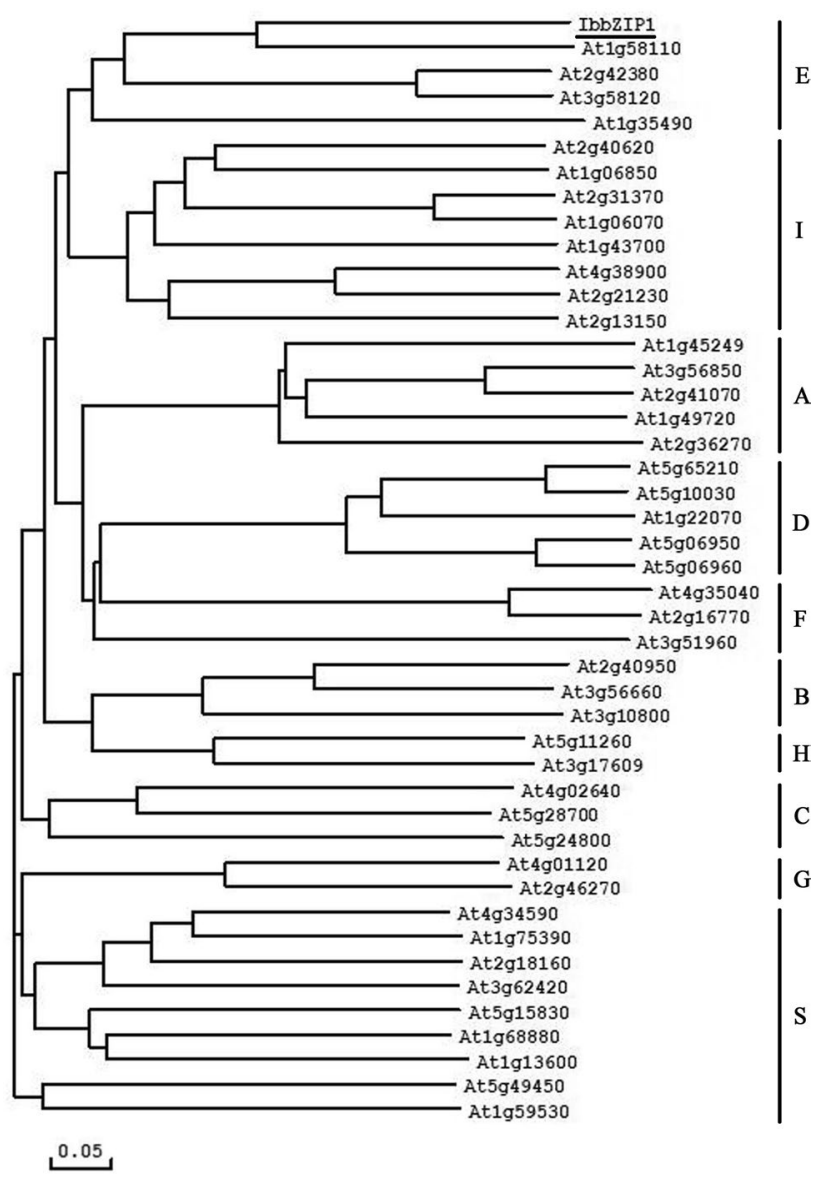

Fig. 1 Phylogenetic analysis of IbbZIP1 with 44 Arabidopsis bZIP proteins. Ten groups of bZIP proteins were defined based on the classification of Arabidopsis bZIP proteins (Jakoby et al. 2002) 


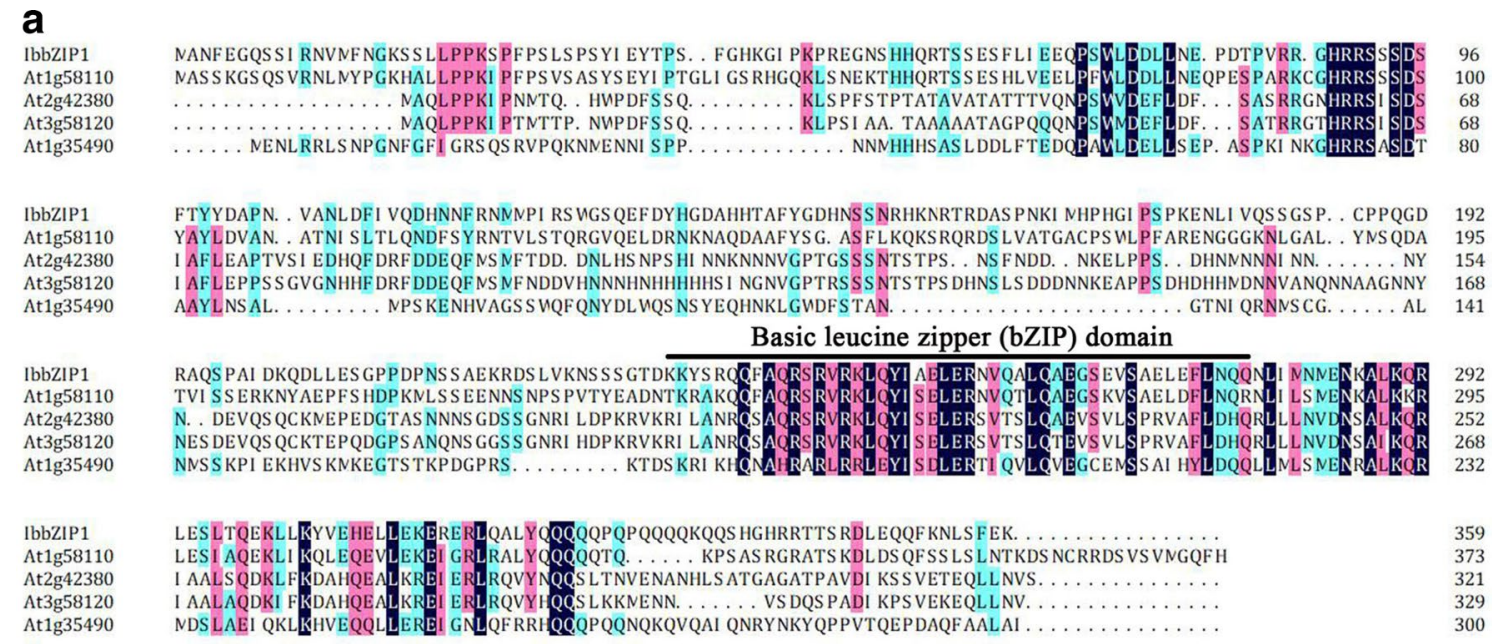

b

IbbZIP1

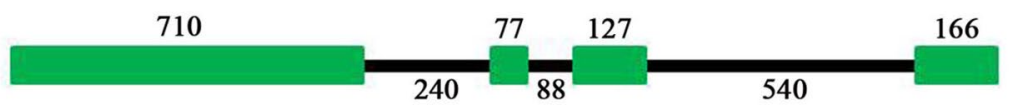

719

76

At1g58110

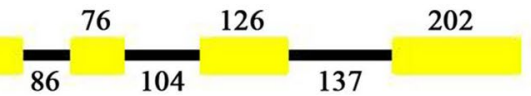

Fig. 2 Comparison between IbbZIP1 and the Arabidopsis E group members. a Sequence alignment of IbbZIP1 with Arabidopsis proteins belonging to the $\mathrm{E}$ group. Black line shows the basic leucine zipper (bZIP) domain. b Comparison of exon and intron constituents

between IbbZIPI and At1g58110. Exons are represented by colorful boxes and introns by black lines, with length (bp) displayed above exons and below introns

\section{Expression of $I b b Z I P 1$ in sweetpotato}

The IbbZIPI gene exhibited significantly higher expression level in the leaves of HVB-3 than in the stems and storage roots (Fig. 3a). Its expression in the in vitro-grown plants of HVB-3 was strongly induced by $\mathrm{NaCl}$, PEG6000 and ABA, and peaked (6.79-fold) at $12 \mathrm{~h}$ of $200 \mathrm{mM} \mathrm{NaCl}$ treatment, (6.58-fold) at $24 \mathrm{~h}$ of $20 \%$ PEG6000 treatment and (7.51fold) at $24 \mathrm{~h}$ of $100 \mu \mathrm{M} \mathrm{ABA}$ treatment (Fig. 3b). These results showed that IbbZIPI might be involved in the tolerance to salt and drought in sweetpotato.

\section{Transactivation activity of IbbZIP1 in yeast}

The yeast one-hybrid system was applied to identify a possible transactivation activity of the IbbZIP1 protein. The yeast cells harboring pGAL4 and pGBKT7-IbbZIPI grew well on SD plate without tryptophan and histidine and exhibited $\beta$-galactosidase activity, but the cells bearing pBD failed to grow (Fig. 4). These results demonstrated that IbbZIP1 had transactivation activity in yeast and has potential ability to activate the transcription of related genes by directly binding to $c i s$-acting elements in their promoter region or interacting with other proteins.

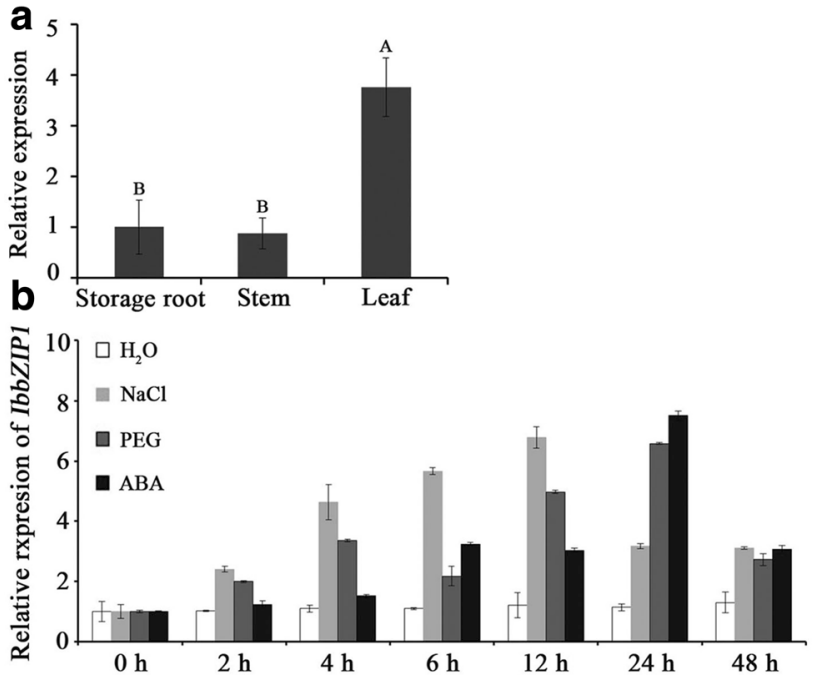

Fig. 3 a Expression analysis of $I b b Z I P 1$ in storage root, stem and leaf tissues of HVB-3. Different capital letters indicate a significant difference at $P<0.01$ by Student's $t$ test. b Expression analysis of the IbbZIP1 gene in the in vitro-grown plants of HVB-3 after different times (h) in response to $\mathrm{H}_{2} \mathrm{O}, 200 \mathrm{mM} \mathrm{NaCl}, 20 \%$ PEG6000 and $100 \mu \mathrm{M}$ ABA, respectively. Data are presented as mean values $\pm \mathrm{SE}$ $(n=3)$ 


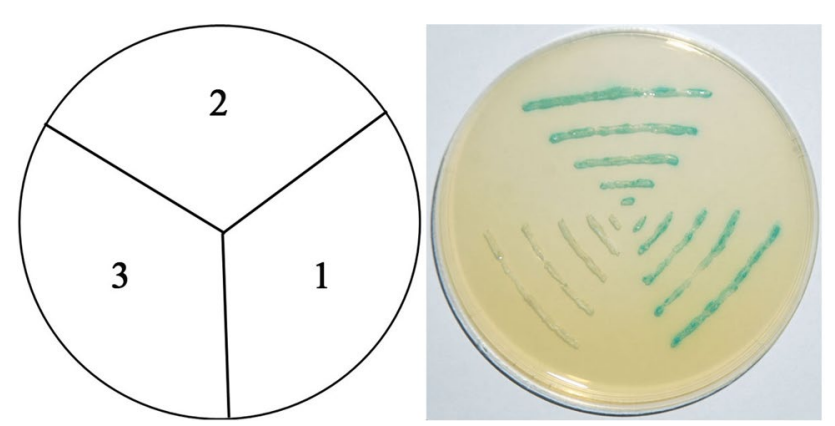

Fig. 4 Transactivation assay of IbbZIP1 in yeast. (1) the pGAL4 vector as positive control. (2) pBD-IbbZIP1. (3) the empty pBD vector as negative control. The culture solution of the transformed yeast was drawn onto the SD plate without tryptophan and histidine

\section{Production of the transgenic Arabidopsis plants}

Lots of putatively transgenic Arabidopsis seeds were obtained by the dipping flower method. The randomly sampled seeds were sown on MS medium with $12.5 \mathrm{mg} / \mathrm{L}$ phosphinothricin (PPT) and produced plants. GUS assay and PCR analysis confirmed that 8 of the randomly sampled 60 plants were transgenic plants, named L1, L2, ... L8, respectively (Supplementary Fig. S2), from which $\mathrm{T}_{3}$ were generated. The expression of IbbZIPI in the leaves of the $\mathrm{T}_{3}$ and WT plants was analyzed by qRT-PCR and 4 of them (L1, L3, L6 and L8) exhibited significantly higher expression level compared with other plants (Supplementary Fig. S2).

\section{Enhanced salt and drought tolerance of transgenic Arabidopsis}

Four transgenic lines (L1, L3, L6, and L8) and WT seedlings were cultured on MS medium with $200 \mathrm{mM} \mathrm{NaCl}$, $300 \mathrm{mM}$ mannitol or without stresses for 2 weeks, respectively. The transgenic and WT plants exhibited no differences in growth without stresses, but the transgenic plants showed significantly better growth and increased physical size than WT under $\mathrm{NaCl}$ and mannitol stresses (Fig. 5).

Two-week-old transgenic and WT plants grown in pots were stressed by $300 \mathrm{mM} \mathrm{NaCl}$ and drought, respectively. The transgenic plants and WT showed no differences in growth without stresses (Fig. 6a). Under salt and drought stresses, the transgenic plants exhibited good growth, increased $\mathrm{ABA}$ and proline contents, enhanced SOD activity and decreased $\mathrm{H}_{2} \mathrm{O}_{2}$ content, while WT almost died (Fig. 6a, b). These results indicated that L1, L3, L6, and L8 had significantly enhanced salt and drought tolerance compared with WT. a

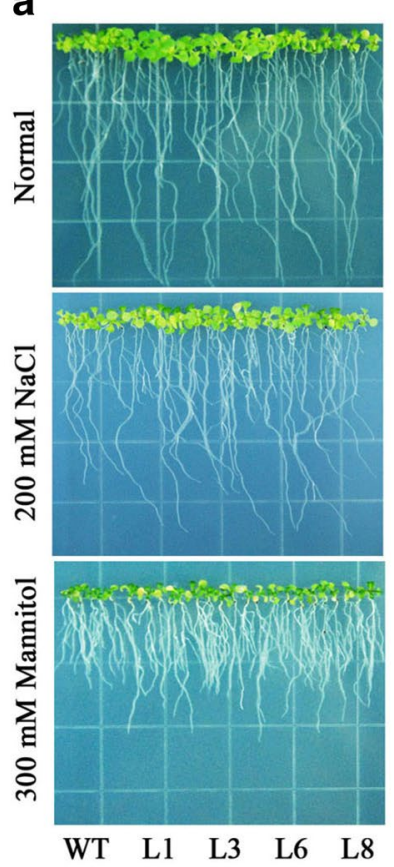

b
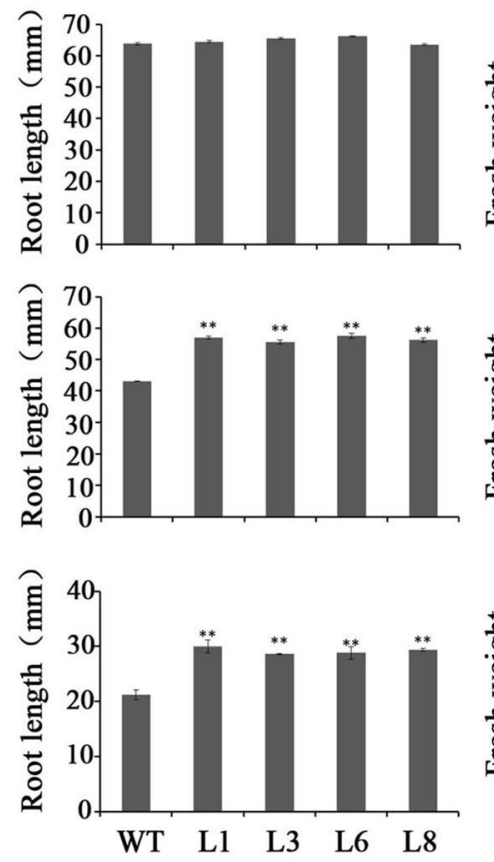
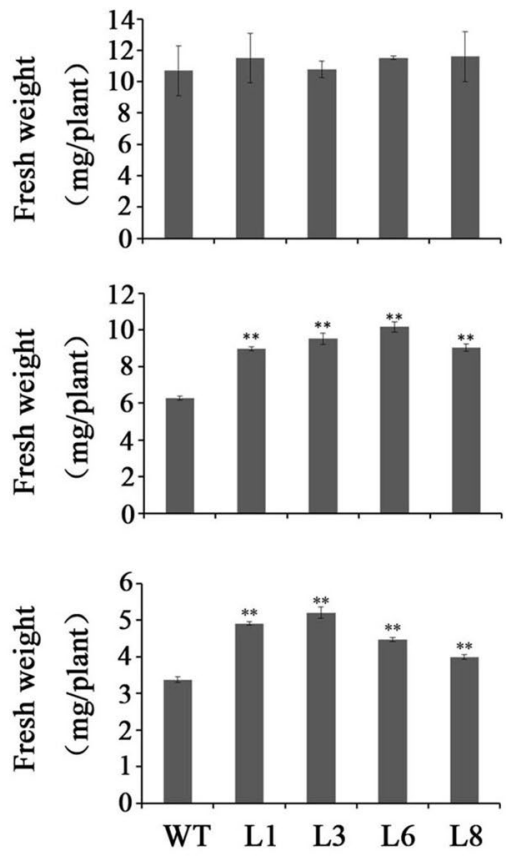

Fig. 5 Responses of the transgenic Arabidopsis seedlings and WT cultured for 2 weeks on MS medium with $200 \mathrm{mM} \mathrm{NaCl}$ and $300 \mathrm{mM}$ mannitol, respectively. a Growth and rooting of the trans- genic Arabidopsis seedlings and WT. b Root length and plant fresh weight of the transgenic Arabidopsis seedlings and WT. Data are presented as mean $\pm \operatorname{SE}(n=3)$ 

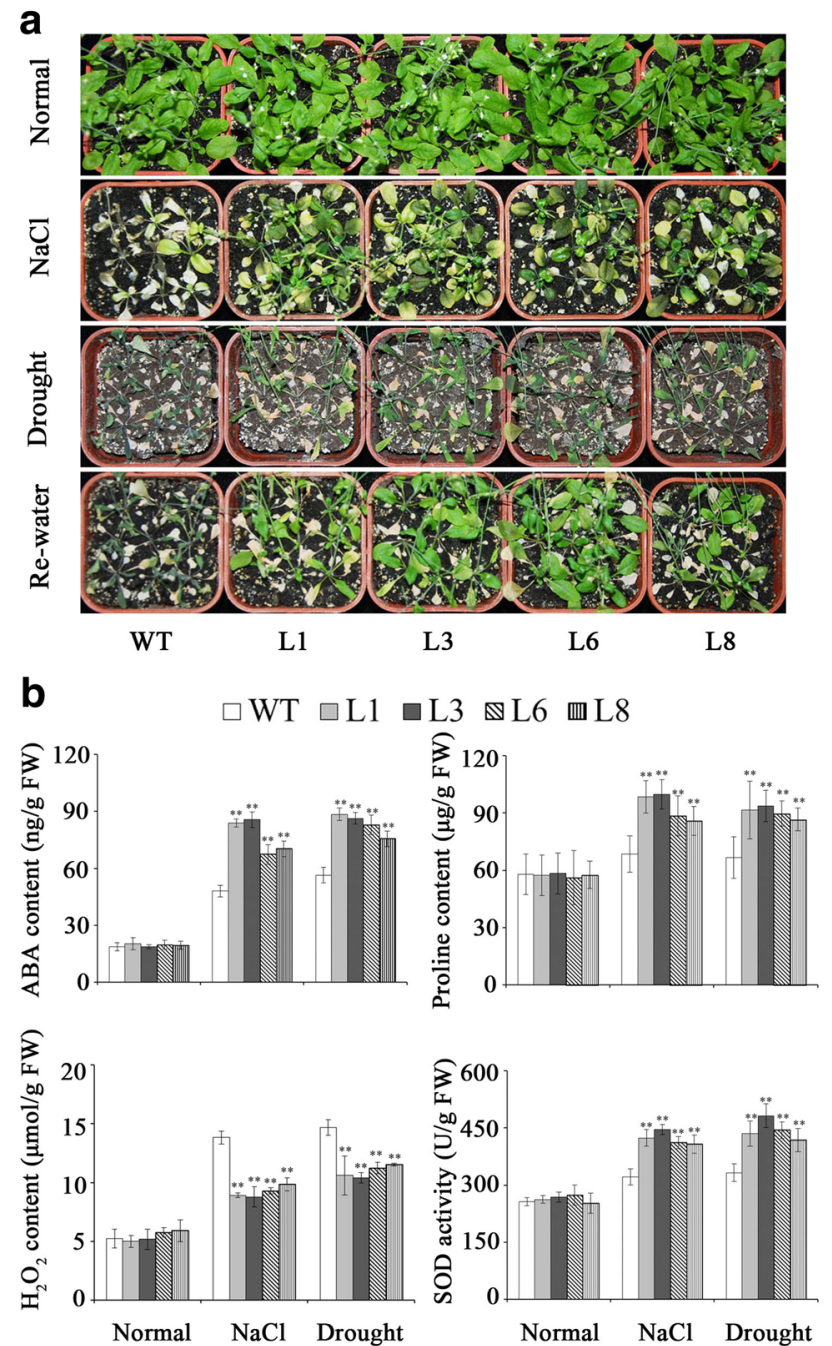

Fig. 6 Responses of the transgenic Arabidopsis plants and WT grown in pots under salt and drought stresses. a Phenotypes of transgenic vs. WT plants grown for 6 weeks under normal condition, 2 weeks under $300 \mathrm{mM} \mathrm{NaCl}$ stress and 4 weeks under drought stress and then 2 days after re-watering, respectively. b ABA, proline, and $\mathrm{H}_{2} \mathrm{O}_{2}$ contents and SOD activity in the transgenic plants and WT grown for 4 weeks under normal condition, 1 weeks under $300 \mathrm{mM} \mathrm{NaCl}$ and 2 weeks under drought stress. Data are presented as mean $\pm \mathrm{SE}(n=3)$

\section{Increased ABA sensitivity of transgenic Arabidopsis}

Seed germination in response to ABA was tested to determine whether IbbZIPl is involved in the ABA signaling pathway. No obvious differences in germination rate and cotyledon opening and greening rate were observed between the transgenic lines (L1, L3, L6, and L8) and WT under normal condition (Fig. 7). With exposure to different concentrations of ABA, both germination rate and cotyledon opening and greening rate of the transgenic and WT seeds declined, but the germination of L1, L3, L6, and L8 seeds were more sensitive to ABA-elicited inhibition, indicating that this gene might be involved in the $\mathrm{ABA}$ signaling pathway (Fig. 7).

\section{Expression of the stress-responsive genes in the transgenic Arabidopsis plants}

The genes encoding ABA biosynthetic 9-cis-epoxycarotenoid dioxygenase (NCED) and xanthoxin dehydrogenase (ABA2) and proline biosynthetic pyrroline-5-carboxylate synthase (P5CS) showed significantly higher expression levels in the transgenic plants in comparison with WT under salt and drought stresses (Fig. 8). The ROS scavenging genes encoding SOD, glutathione peroxidase (GPX), catalase (CAT), ascorbate peroxidase (APX) and dehydroascorbate reductase (DHAR) were found to show a systematic upregulation in the transgenic plants under salt and drought stresses (Fig. 8).

\section{Discussion}

Several bZIP transcription factor genes have been cloned from Arabidopsis, rice, soybean, maize etc., and found to play roles in plant stress-responsive and hormone signal transduction (Sun et al. 2012; Liu et al. 2014a; Gao et al. 2011a; Wang et al. 2017). However, there is no report on the bZIP transcription factors in sweetpotato. This study reported, for the first time, the cloning and characterization of a novel transcription factor gene, IbbZIPI, from sweetpotato. The IbbZIP1 protein contains a typical bZIP domain which is essential for bZIP transcription factor, belongs to E group of bZIP family and has a close relationship with At1g58110 (Figs. 1, 2). To date, the function of the Atlg58110 gene is still unknown. We found that the promoter region of IbbZIPI had the stress-responsive cisacting regulatory elements (Yamaguchi-Shinozaki et al. 2005, 2006; Xiang et al. 2008). The IbbZIP1 expression was induced by $\mathrm{NaCl}$, PEG, and ABA (Figs. S1, 4; Supplementary Table S2) and its overexpression increased salt and drought tolerance in transgenic Arabidopsis (Figs. 6, 7).

The bZIP transcription factors regulate the ABA-mediated abiotic stresses signaling pathways in plants (Mehrotra et al. 2014). In rice, overexpression of $O S A B I 5$ and OsbZIP23 upregulated the ABA biosynthetic gene $A B A 2$ (Zou et al. 2008; Xiang et al. 2008). The ABA biosynthetic gene $N C E D 2$ was upregulated in the $Z m A B P 9$-overexpressing cotton plants (Wang et al. 2017). ABA regulates the expression of ABA dependent stress-responsive genes and higher levels of ABA can enhance salt and drought tolerance in Arabidopsis (Tuteja 2007; Liu et al. 2013; Wang et al. 2016a). In the present study, increased ABA sensitivity of transgenic Arabidopsis to exogenous ABA showed that IbbZIPI might be involved in the ABA signaling pathway 


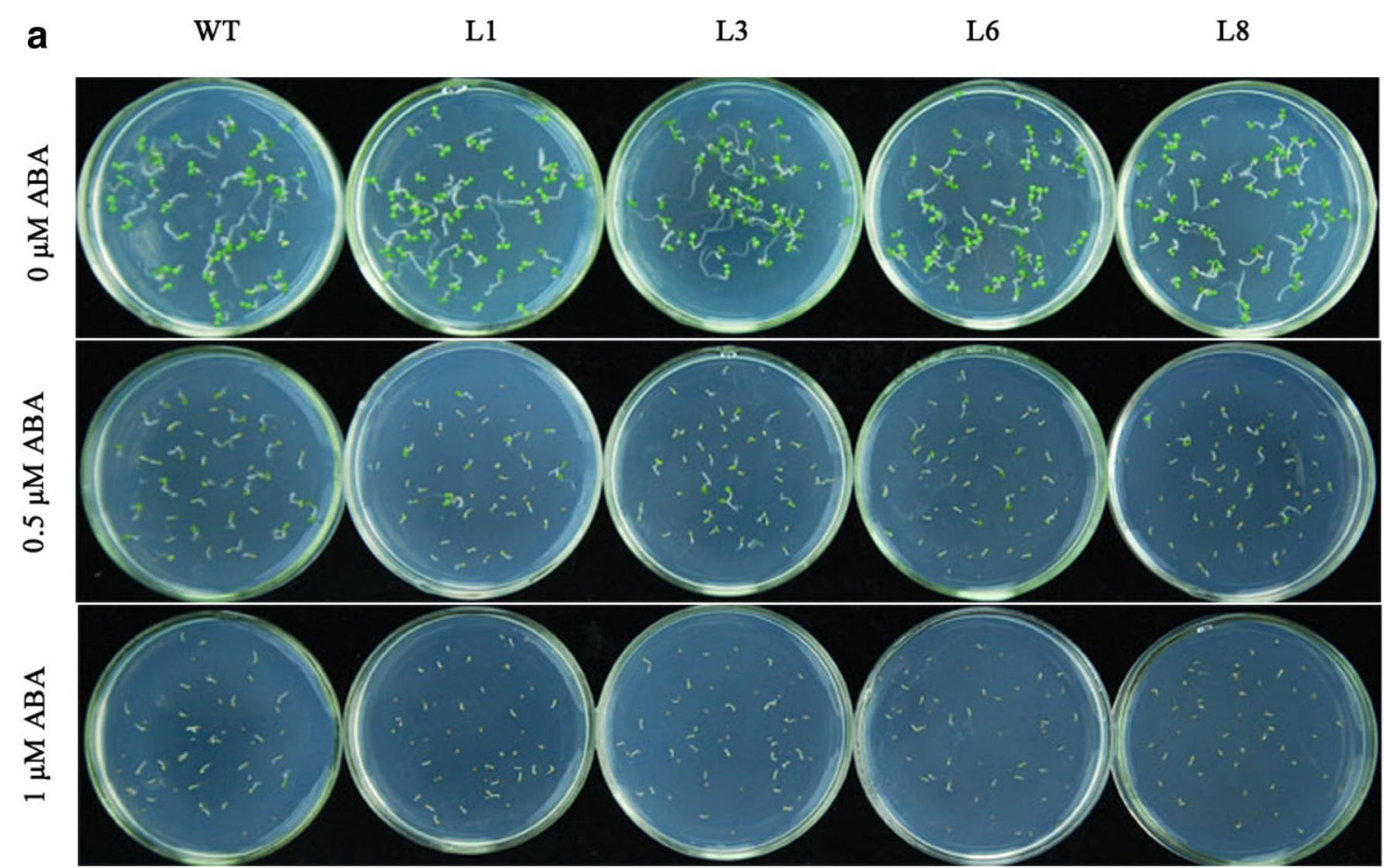

b

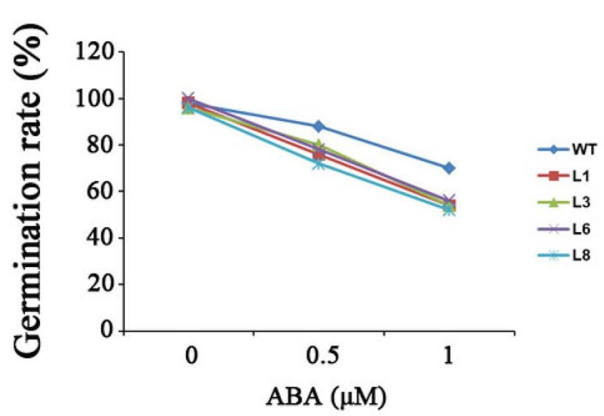

Fig. 7 Responses of the transgenic Arabidopsis seeds and WT sown on MS medium with $0,0.5$, and $1 \mu \mathrm{M}$ ABA for 1 week. a Growth vigor of the transgenic and WT seedlings. b Germination rates of

(Fig. 7). The IbbZIP1-overexpressing Arabidopsis plants exhibited significant upregulation of $N C E D$ and $A B A 2$ and significant increase of ABA content under salt and drought stresses (Figs. 7, 8). It is suggested that overexpression of IbbZIP I confers tolerance to salt and drought due to the increased expression level of the ABA biosynthetic genes, which increases the production of ABA as a signaling molecule and further the expression of stress-responsive genes.

It has been reported that the high level of ABA in rice increases the transcript level of OSP5CS1, which lead to accumulation of proline under abiotic stresses (Sripinyowanich et al. 2013). It has been shown that proline accumulation resulted in the enhanced salt and drought tolerance in several plant species (Szabados and Savouré 2010; Krasensky and Jonak 2012; Zhang et al. 2012; Liu et al. 2015). Overexpression of GmbZIP62 and GmbZIP78 upregulated the
C

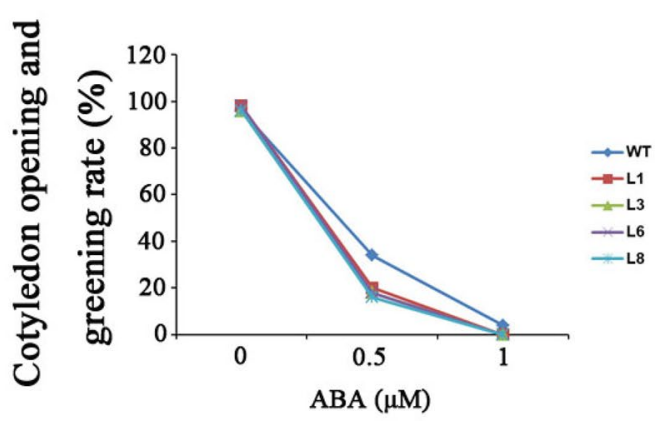

the transgenic Arabidopsis seeds and WT. c Cotyledon opening and greening rates of the transgenic Arabidopsis seeds and WT

genes involved in ABA signaling pathways and GmP5CS1, resulting the increased proline content and the enhanced salt and freezing tolerance in transgenic Arabidopsis (Liao et al. 2008). In this study, the IbbZIPI-overexpressing Arabidopsis plants exhibited the increased $P 5 C S$ expression level and proline content under salt and drought stresses (Figs. 7, 8). These results suggested that more proline might be accumulated because of the increased ABA level, which leads to the enhanced salt and drought tolerance.

In plants, overproduction of ROS often occurs under salinity and drought stresses, which leads to oxidative damage. ROS can be detoxified by activating ROS scavenging enzymes (Gill and Tuteja 2010). The increased proline level led to upregulation of the ROS scavenging genes, which resulted in the enhanced tolerance to salt and drought in transgenic sweetpotato (Liu et al. 2014b, 


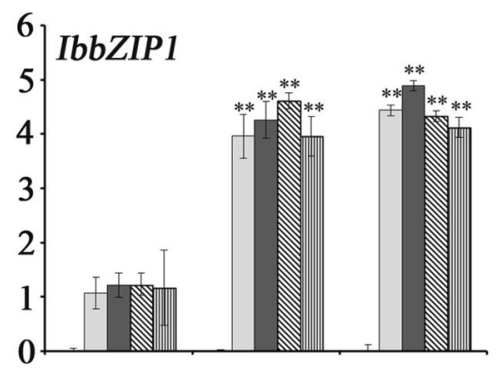

$\square \mathrm{WT} \square \mathrm{L} 1 \square \mathrm{L} 3 \mathbb{\mathrm { L }} 6 \quad \mathbf{m} \mathrm{L} 8$
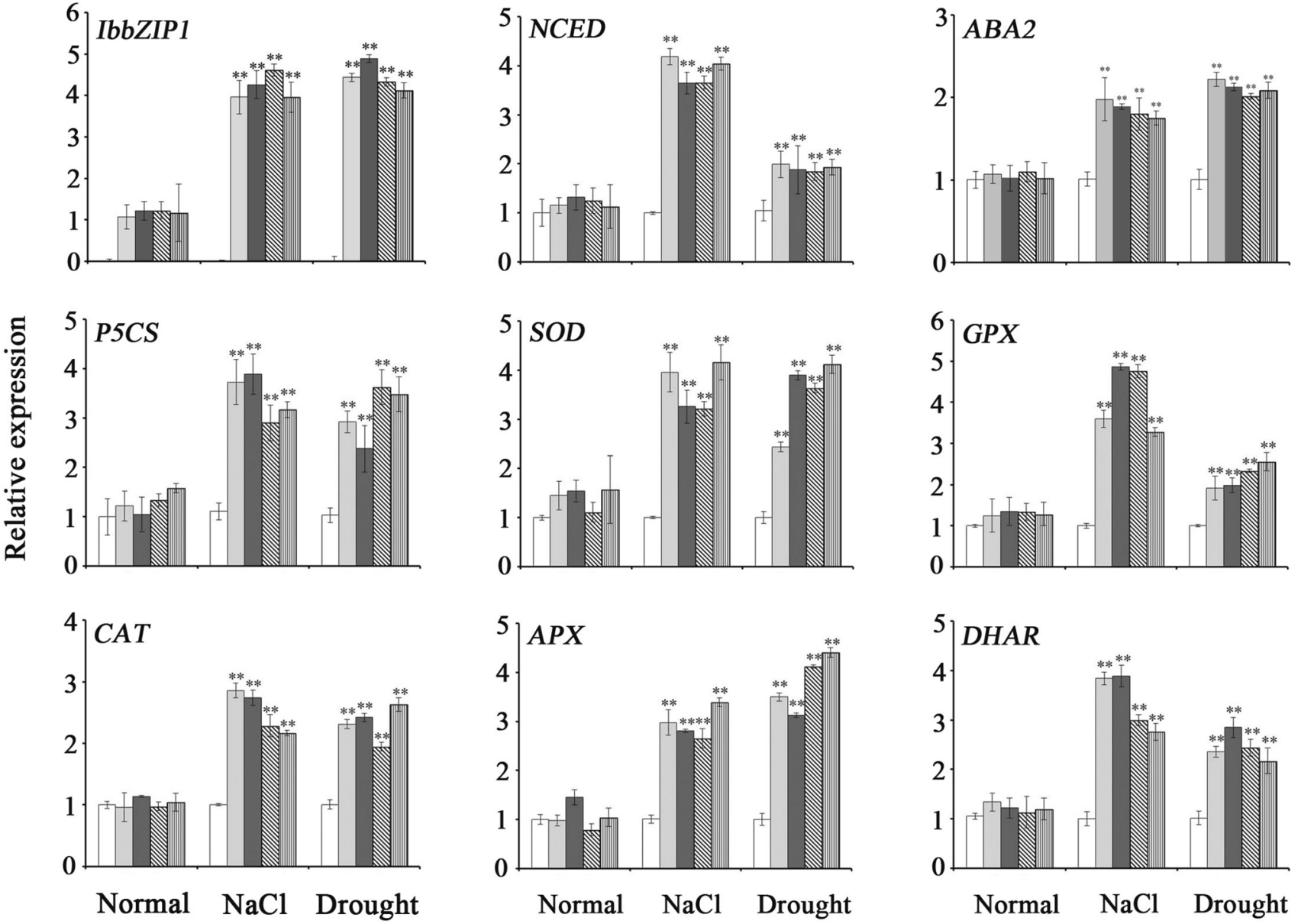

Fig. 8 Transcript levels of salt- and drought-responsive genes in the transgenic Arabidopsis plants and WT under salt and drought stresses. Leaves of transgenic and WT pot-grown plants incubated for
4 weeks under normal condition, 2 weeks under $300 \mathrm{mM} \mathrm{NaCl}$ and 4 weeks under drought stress were used for expression analysis of the genes. Data are presented as mean $\pm \operatorname{SE}(n=3)$
Liu et al. 2015; Wang et al. 2016b; Zhai et al. 2015). We found that under salt and drought stresses, the ROS scavenging genes (SOD, GPX, CAT, APX, and DHAR) were systematically upregulated in the IbbZIPI-overexpressing Arabidopsis plants (Fig. 8). Thus, more accumulation of proline in the IbbZIPI-overexpressing Arabidopsis plants might upregulate the ROS scavenging genes and further stimulate the ROS scavenging system, which results in the enhanced salt and drought tolerance.

In conclusion, a novel sweetpotato bZIP transcription factor gene, IbbZIP1, has been successfully isolated. The $I b b Z I P 1$ gene is involved in salt and drought tolerance in transgenic Arabidopsis. Its overexpression might upregulate the genes involved in ABA and proline biosynthesis and ROS scavenging system and increase the ABA and proline contents, which leads to enhanced salt and drought tolerance. This study provides a novel bZIP gene for improving the tolerance of sweetpotato and other plants to abiotic stresses.

Author contribution statement Conceived and designed the experiments: QCL CK. Performed the experiments: CK. Analyzed the data: CK. Contributed reagents/materials/analysis tools: QCL HZ SZH NZ. Wrote the paper: QCL CK.

Acknowledgements This work was supported by National Natural Science Foundation of China (31461143017) and China Agriculture Research System (CARS-10, Sweetpotato).

\section{Compliance with ethical standards}

Conflict of interest The authors declare that they have no conflict of interest. 
Open Access This article is distributed under the terms of the Creative Commons Attribution 4.0 International License (http://creativeco mmons.org/licenses/by/4.0/), which permits unrestricted use, distribution, and reproduction in any medium, provided you give appropriate credit to the original author(s) and the source, provide a link to the Creative Commons license, and indicate if changes were made.

\section{References}

Bohnert HJ, Nelson DE, Jensen RG (1995) Adaptations to environmental stresses. Plant Cell 7:1099-1111

Clough SJ, Bent AF (1998) Floral dip: a simplified method for Agrobacterium-mediated transformation of Arabidopsis thaliana. Plant J 16:735-743

Gao S, Yuan L, Zhai H, Liu CL, He SZ, Liu QC (2011a) Transgenic sweetpotato plants expressing an LOS5 gene are tolerant to salt stress. Plant Cell, Tissue Organ Cult 107:205-213

Gao SQ, Chen M, Xu ZS, Zhao CP, Li LC, Xu HJ, Tang YM, Zhao X, Ma YZ (2011b) The soybean GmbZIP1 transcription factor enhances multiple abiotic stress tolerances in transgenic plants. Plant Mol Biol 75:537-553

Gill SS, Tuteja N (2010) Reactive oxygen species and antioxidant machinery in abiotic stress tolerance in crop plants. Plant Physiol Biochem 48:909-930

Hossain MA, Cho JI, Han M, Ahn CH, Jeon JS, An G, Park PB (2010a) The ABRE-binding bZIP transcription factor $O s A B F 2$ is a positive regulator of abiotic stress and ABA signaling in rice. J Plant Physiol 167:1512-1520

Hossain MA, Lee Y, Cho JI, Ahn CH, Lee SK, Jeon JS, Kang H, Lee CH, An G, Park PB (2010b) The bZIP transcription factor $O s A B F 1$ is an ABA responsive element binding factor that enhances abiotic stress signaling in rice. Plant Mol Biol 72:557-566

Jakoby M, Weisshaar B, Dröge-Laser W, Vicente-Carbajosa J, Tiedemann J, Kroj T, Parcy F (2002) bZIP transcription factors in Arabidopsis. Trends Plant Sci 7:106-111

Jefferson RA, Kavanagh TA, Bevan MW (1987) GUS fusions: $\beta$-glucuronidase as a sensitive and versatile gene fusion marker in higher plants. EMBO J 6:3901-3907

Jiang XQ, Zhang CQ, Lü PT, Jiang GM, Liu XW, Dai FW, Gao JP (2014) RhNAC3, a stress-associated NAC transcription factor, has a role in dehydration tolerance through regulating osmotic stressrelated genes in rose petals. Plant Biotechnol J 12:38-48

Kim JB, Kang JY, Kim SY (2004) Over-expression of a transcription factor regulating ABA-responsive gene expression confers multiple stress tolerance. Plant Biotechnol J 2:459-466

Kim SH, Ahn YO, Ahn MJ, Lee HS, Kwak SS (2012) Down-regulation of $\beta$-carotene hydroxylase increases $\beta$-carotene and total carotenoids enhancing salt stress tolerance in transgenic cultured cells of sweetpotato. Phytochemistry 74:69-78

Kim SH, Kim YH, Ahn YO, Ahn MJ, Jeong JC, Lee HS, Kwak SS (2013) Downregulation of the lycopene $\varepsilon$-cyclase gene increases carotenoid synthesis via the $\beta$-branch-specific pathway and enhances salt-stress tolerance in sweetpotato transgenic calli. Physiol Plant 147:432-442

Kim SH, Jeong JC, Park S, Bae JY, Ahn MJ, Lee HS, Kwak SS (2014) Down-regulation of sweetpotato lycopene $\beta$-cyclase gene enhances tolerance to abiotic stress in transgenic calli. Mol Biol Rep 41:8137-8148

Krasensky J, Jonak C (2012) Drought, salt, and temperature stressinduced metabolic rearrangements and regulatory networks. J Exp Bot 63:1593-1608

Li RJ, Kang C, Song XJ, Yu L, Liu DG, He SZ, Zhai H, Liu QC (2017) A $\zeta$-carotene desaturase gene, IbZDS, increases $\beta$-carotene and lutein contents and enhances salt tolerance in transgenic sweetpotato. Plant Sci 262:39-51

Liao Y, Zou H, Wei W, Hao YJ, Tian AG, Huang J, Liu YF, Zhang JS, Chen SY (2008) Soybean GmbZIP44, GmbZIP62 and GmbZIP78 genes function as negative regulator of ABA signaling and confer salt and freezing tolerance in transgenic Arabidopsis. Planta 228:225-240

Lindemose S, O'Shea C, Jensen MK, Skriver K (2013) Structure, function and networks of transcription factors involved in abiotic stress responses. Int J Mol Sci 14:5842-5878

Liu CT, Wu YB, Wang XP (2012) bZIP transcription factor OsbZIP52/RISBZ5: a potential negative regulator of cold and drought stress response in rice. Planta 235:1157-1169

Liu XX, Zhai SM, Zhao YJ, Sun BC, Liu C, Yang AF, Zhang JR (2013) Overexpression of the phosphatidylinositol synthase gene $(Z m P I S)$ conferring drought stress tolerance by altering membrane lipid composition and increasing ABA synthesis in maize. Plant, Cell Environ 36:1037-1055

Liu CT, Mao BG, Ou SJ, Wang W, Liu LC, Wu YB, Chu CC, Wang XP (2014a) OsbZIP71, a bZIP transcription factor, confers salinity and drought tolerance in rice. Plant Mol Biol 84:19-36

Liu DG, He SZ, Zhai H, Wang LJ, Zhao Y, Wang B, Li RJ, Liu QC (2014b) Overexpression of IbP5CR enhances salt tolerance in transgenic sweetpotato. Plant Cell, Tissue Organ Cult 117:1-16

Liu DG, Wang LJ, Liu CL, Song XJ, He SZ, Zhai H, Liu QC (2014c) An Ipomoea batatas iron-sulfur cluster scaffold protein gene, IbNFU1, is involved in salt tolerance. PLoS ONE 9:e93935

Liu DG, Wang LJ, Zhai H, Song XJ, He SZ, Liu QC (2014d) A novel a/b-hydrolase gene IbMas enhances salt tolerance in transgenic sweetpotato. PLoS ONE 9:e115128

Liu DG, He SZ, Song XJ, Zhai H, Liu N, Zhang DD, Ren ZT, Liu QC (2015) IbSIMT1, a novel salt-induced methyltransferase gene from Ipomoea batatas, is involved in salt tolerance. Plant Cell, Tissue Organ Cult 120:701-715

Lu GJ, Gao CX, Zheng XN, Han B (2009) Identification of OsbZIP72 as a positive regulator of $\mathrm{ABA}$ response and drought tolerance in rice. Planta 229:605-615

Mehrotra R, Bhalothia P, Bansal P, Basantani MK, Bharti V, Mehrotra S (2014) Abscisic acid and abiotic stress tolerance-different tiers of regulation. J Plant Physiol 171:486-496

Munns R, Tester M (2008) Mechanisms of salinity tolerance. Annu Rev Plant Biol 59:651-681

Schmittgen TD, Livak KJ (2008) Analyzing real-time PCR data by the comparative $C_{\mathrm{T}}$ method. Nat Protoc 3:1101-1108

Sripinyowanich S, Klomsakul P, Boonburapong B, Bangyeekhun T, Asami T, Gu HY, Buaboocha T, Chadchawan S (2013) Exogenous ABA induces salt tolerance in indica rice (Oryza sativa L.): the role of OsP5CS1 and OsP5CR gene expression during salt stress. Environ Exp Bot 86:94-105

Sun XL, Li Y, Cai H, Bai X, Ji W, Ding XD, Zhu YM (2012) The Arabidopsis AtbZIP1 transcription factor is a positive regulator of plant tolerance to salt, osmotic and drought stresses. J Plant Res 125:429-438

Szabados L, Savouré A (2010) Proline: a multifunctional amino acid. Trends Plant Sci 15:89-97

Tuteja N (2007) Abscisic acid and abiotic stress signaling. Plant Signal Behav 2:135-138

Wang B, Zhai H, He SZ, Zhang H, Ren ZT, Zhang DD, Liu QC (2016a) A vacuolar $\mathrm{Na}+/ \mathrm{H}+$ antiporter gene, $I b N H X 2$, enhances salt and drought tolerance in transgenic sweetpotato. Sci Hortic 201:153-166

Wang FB, Tong WJ, Zhu H, Kong WL, Peng RH, Liu QC, Yao QH (2016b) A novel Cys2/His2 zinc finger protein gene from sweetpotato, IbZFP1, is involved in salt and drought tolerance in transgenic Arabidopsis. Planta 243:783-797 
Wang FB, Zhai H, An YY, Si ZZ, He SZ, Liu QC (2016c) Overexpression of IbMIPS1 gene enhances salt tolerance in transgenic sweetpotato. J Integr Agric 15:271-281

Wang CL, Lu GQ, Hao YQ, Guo HM, Guo Y, Zhao J, Cheng HM (2017) ABP9, a maize bZIP transcription factor, enhances tolerance to salt and drought in transgenic cotton. Planta 246:453-469

Xiang Y, Tang N, Du H, Ye H, Xiong LZ (2008) Characterization of OsbZIP23 as a key player of the basic leucine zipper transcription factor family for conferring abscisic acid sensitivity and salinity and drought tolerance in rice. Plant Physiol 148:1938-1952

Yamaguchi-Shinozaki K, Shinozaki K (2005) Organization of cis-acting regulatory elements in osmotic- and cold-stress-responsive promoters. Trends Plant Sci 10:88-94

Yamaguchi-Shinozaki K, Shinozaki K (2006) Transcriptional regulatory networks in cellular responses and tolerance to dehydration and cold stresses. Ann Rev Plant Biol 57:781-803

Yang O, Popova OV, Süthoff U, Lüking I, Dietz K, Golldack D (2009) The Arabidopsis basic leucine zipper transcription factor AtbZIP24 regulates complex transcriptional networks involved in abiotic stress resistance. Gene 436:45-55

Yang SJ, Vanderbeld B, Wan JX, Huang YF (2010) Narrowing down the targets: towards successful genetic engineering of droughttolerant crops. Mol Plant 3:469-490

Ying S, Zhang DF, Fu J, Shi YS, Song YC, Wang TY, Li Y (2012) Cloning and characterization of a maize bZIP transcription factor, ZmbZIP72, confers drought and salt tolerance in transgenic Arabidopsis. Planta 235:453-469
Zang N, Zhai H, Gao S, Chen W, He SZ, Liu QC (2009) Efficient production of transgenic plants using the bar gene for herbicide resistance in sweetpotato. Sci Hortic 122:649-653

Zhai H, Wang FB, Si ZZ, Huo JX, Xing L, An YY, He SZ, Liu QC (2015) A myo-inositol-1-phosphate synthase gene, IbMIPS1, enhances salt and drought tolerance and stem nematode resistance in transgenic sweet potato. Plant Biotechnol J 14:592-602

Zhang H, Han B, Wang T, Chen SX, Li HY, Zhang YH, Dai SJ (2012) Mechanisms of plant salt response: insights from proteomics. J Proteome Res 11:49-67

Zhao Q, Zhang H, Wang T, Chen S, Dai SJ (2013) Proteomics-based investigation of salt-responsive mechanisms in plant roots. J Proteoeme 82:230-253

Zhu JK (2001) Plant salt tolerance. Trends Plant Sci 6:66-71

Zhu JK (2002) Salt and drought stress signal transduction in plants. Annu Rev Plant Biol 53:247-273

Zou MJ, Guan YC, Ren HB, Zhang F, Chen F (2008) A bZIP transcription factor, $O S A B I 5$, is involved in rice fertility and stress tolerance. Plant Mol Biol 66:675-683

Publisher's Note Springer Nature remains neutral with regard to jurisdictional claims in published maps and institutional affiliations. 